Research Article

Gabriel Fedorko*, Martin Vasil, and Bibiana Podracka

\title{
A simulation model for the need for intra-plant transport operation planning by AGV
}

https://doi.org/10.1515/eng-2019-0083

Received Aug 18, 2019; accepted Oct 10, 2019

\begin{abstract}
Intra-plant transport has an important role in the systems of enterprise logistics. At present, automated transport systems (AGV) are used for its efficient operation, with minimal operator attendance. For the proper and reliable functioning of such a transport system, there is currently a wide range of methods, of which the method of computer simulation is increasingly dominant. In the application, however, it is necessary to take into account that the functioning of AGV systems is a very demanding process with high demands on the used simulation software. Within the article, it will be described the use of the method of additional programming, as an effective tool in the creation of a simulation model of the AGV system for the need of its planning. The model presents the possibilities of increasing of the output of the analyzed production process to more than $70 \%$, and at the same time it indicates insufficient use of workplaces that in one case reaches the value about $10 \%$. The application of this model pointed to the reduction of the number of workplaces by one and with this related increase of output of other workplace.
\end{abstract}

Keywords: AGV, simulation, intralogistics, transport, planning

\section{Introduction}

Automated Guided Vehicles (AGV) are currently a part of many technological units, where they significantly contribute to the efficiency of ongoing processes. Different methods and approaches are used by their design and planning, for example, fuzzy logic and genetic algo-

\footnotetext{
*Corresponding Author: Gabriel Fedorko: Technical University of Kosice, Faculty BERG, Institute of Logistics and Transport, Park Komenskeho 9, 04001 Kosice, Slovak Republic; Email: gabriel.fedorko@tuke.sk

Martin Vasil, Bibiana Podracka: Technical University of Kosice, Faculty BERG, Institute of Logistics and Transport, Park Komenskeho 9, 04001 Kosice, Slovak Republic
}

Ә Open Access. ( 2019 G. Fedorko et al., published by De Gruyter. (cC) BY 4.0 License rithms [1]. They are also used for the solution of tasks associated with for example with coordination of operation [2] or orientation of transport route [3]. Mathematical models are mainly used in the design of the effective operation of AGV systems [4]. The operation of AGV systems must often be coordinated with business planning [5]. For this reason, it is needed to take into account information from ERP systems [6].

Another important criterion associated with the operation of AGV within intra-plant logistics is the harmonization of their activities with various handling devices [7]. However, the application computer simulation tools and also tools of computer simulation have an important role, for example, application of the method Monte Carlo [8]. For the application of computer simulation within the operation of AGV systems, it is necessary to emphasize the used simulation software [9-11]. This is because such demanding simulation models cannot handle all available simulation programs. At present, the software Tecnomatix Plant Simulation presents an exceptionally suitable program of which application will be presented by the help of a specific example, with using the method of additional programming by the program SimTalk.

The method of additional programming within the program Tecnomatix Plant Simulation has a wide application in the area of AGV. Fedorko et al. [12] used this method to simulate container handling with AGV. Within this research they applied specific programming commands in the language Simtalk, which enabled detailed creation of the simulation model and realization of subsequent experiments and analyses. This reflect the fact that the program Tecnomatix Plant simulation has specifically oriented blocks and commands that are designed for the area of AGV. At the same time, they directly support the use and support of complementary programming [13]. In general, it is possible to state, that the simulation approach in the area of AGV presents an effective tool for the field of analysis and optimization of transport chains by application of transport systems on the base of AGV [14-16]. 


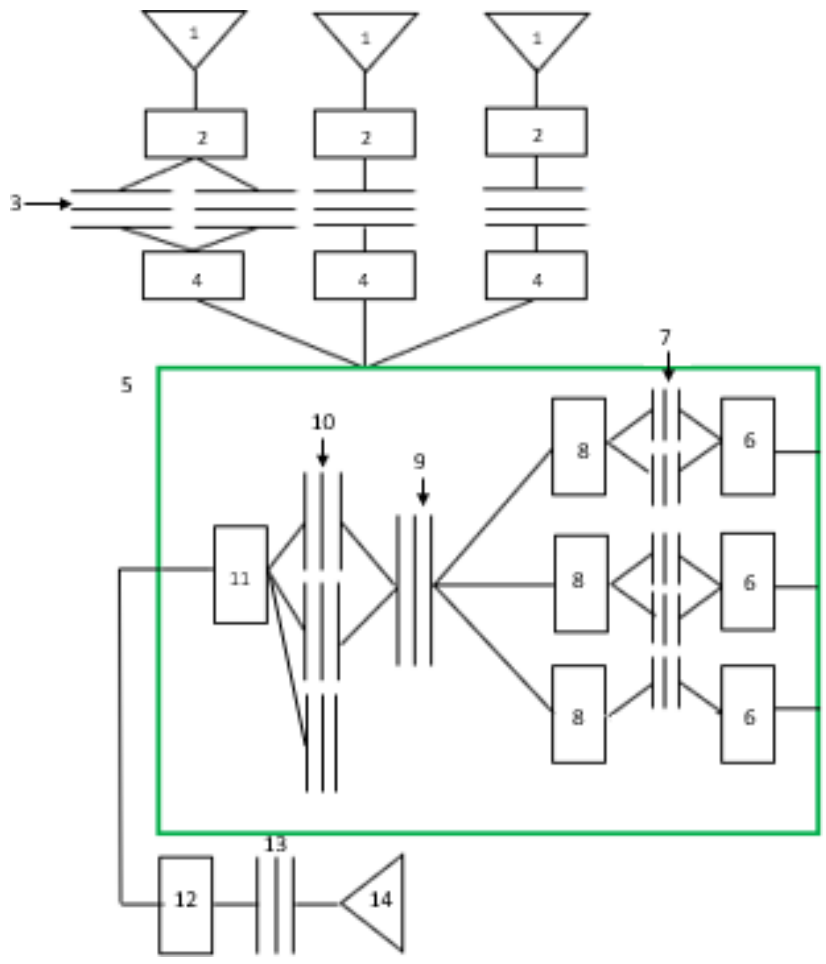

1 - input gate, 2 - delivery entry, 3 - unloading of delivery, 4 - import, 5 - route of the logistics train, 6 - shelf, 7 - machining of components, 8 - shelf, 9 - assembly of components, 10 - end of line, 11 - shelf, 12 - export, 13 - loading, 14 - output gate

Figure 1: Bloch scheme of the simulation model

\section{Characteristics of the simulated process}

The simulation model (Figure 1) presents the production of gearboxes and consists of three main parts. The model also includes a logistics train for supplying of workplaces.

The first main part of the model is the input store which consists of input gates for individual components. It also consists of unloading and import zones from which the components go into production.

The second main part of the simulation model is presented by production with workplaces with machining centres. An important part of production supplying is the logistics train which supplies all workplaces in the model. It is also used to transfer finished products from production to the third main part of the simulation model.

Parts of the gearbox are transported from the input store by logistics train to individual workplaces where they are processed. Subsequently, the machined parts are transferred in front of the assembly line where complete gearboxes are mounted. At the end of the assembly line "end

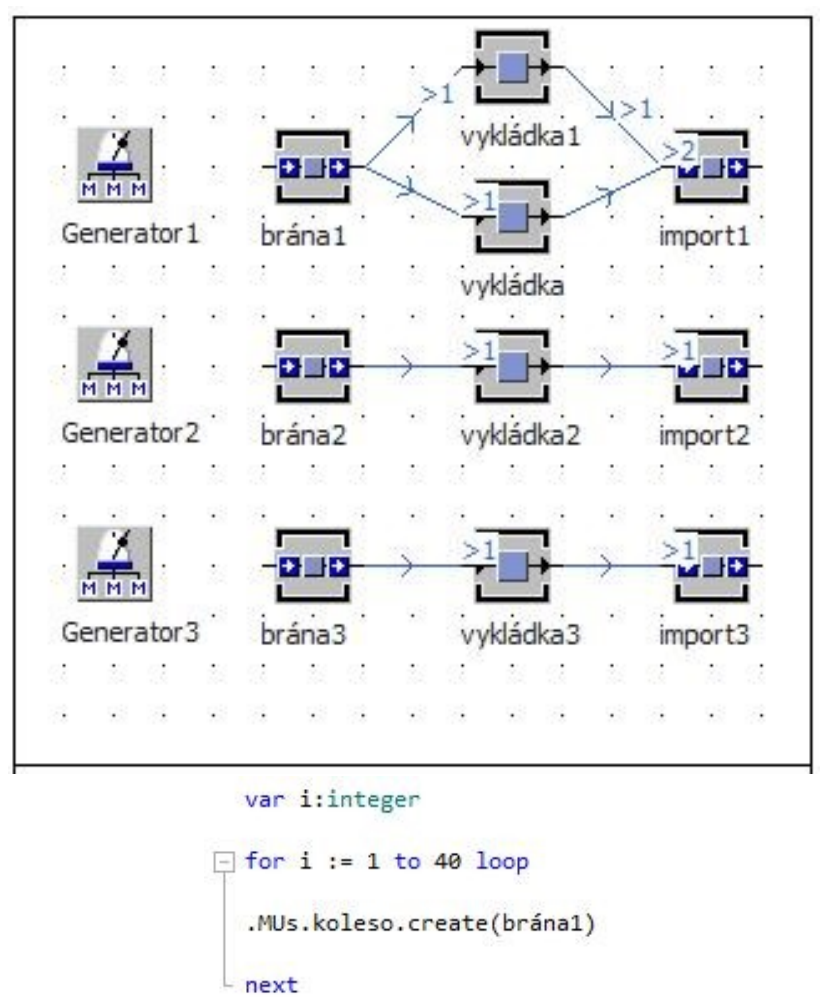

Figure 2: Example of the input store and method for delivery of gear-wheels

of the line", the functionality of the finished gearboxes are checked. The output store is the last part of the simulation model. By the help of the logistics train, the finished products are transferred from the output store, where they are stored and waiting for export.

The part of the simulation model, with the title input store, presents the material entry to the enterprise (Figure 2). The components are supplied from different suppliers within fixed periods. The final product consists of three parts, which present gear-wheels, shafts and gearboxes in the simulation model.

The block named Generator 1 presents the supplier that supplies gear-wheels. This supplier supplies gearwheels at 10 minutes interval. Using additional programming by SimTalk it was created a method for definition of the number of wheels in one batch. (Figure 2). The method includes a cycle with the statement "MUs.wheel.create(gate1)" which will generate a defined number of wheels for the gate 1 .

Generator 2 presents the supplier that supplies the shafts. Because for the assembly of one gear-box we need less number of shafts as wheels, the interval of delivery of the same number of shafts is extended. 
Generator 3 presents the third supplier who supplies gear-boxes. The principle of delivery is the same as that of previous suppliers.

The first step is to unload the components from trucks. Unloading can be realized by employee, forklift or conveyor. In this model, this process is presented by the object Singleproc (unloading, unloading 1, unloading 2 and unloading 3$)$.

Unloading of all three components has a different duration. The time of unloading 1 of gear-wheels is $80 \mathrm{sec}-$ onds. Because it is needed to unload the largest amount of the component in comparison to the others, it was needed to add another employee for unloading of gear-wheels for the balanced and optimized supply of products to the production of all three components. Duration of unloading 2, with the unloading of shafts from the gate 2 , is $120 \mathrm{sec}$ ond. Duration of unloading 3 is 60 second. Unloading 3 presents unloading of gear-boxes. Subsequently, the components are stored and ready for transfer to production.

\subsection{Logistics train}

The logistics train is used in most production enterprises and operates on the principle of components loading in the input store and unloading at individual workplaces in the production. The effort is to maximize the capacity of the truck. There are places of loading import 1, import 2 and import 3 and defined places of unloading shelf 1 , shelf 2 and shelf 3 in this model. The logistics train (Figure 3 ) directs along its route to the shelf 4 , where it loads the finished products and moves them to the exit store.

Furthermore, the logistics train (Figure 3) is directed along its route to the rack4, where it loads the finished products and moves them to the exit store.

The train has default capacity and load capacity. The towing vehicle is the towing vehicle to which the transporters are connected. The capacity of one transporter is

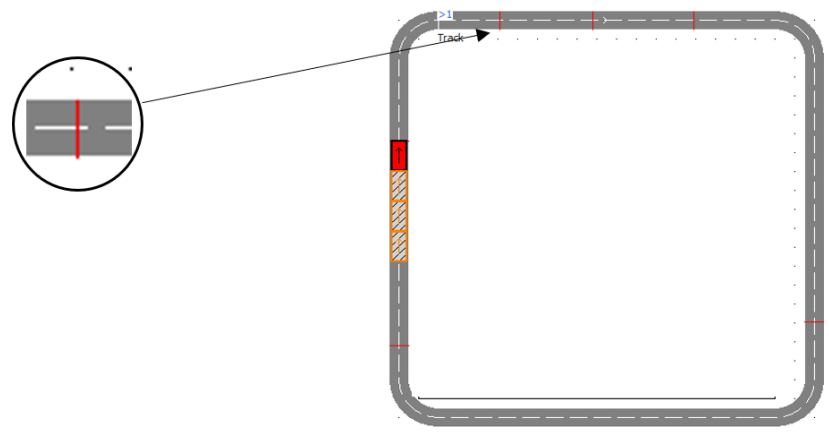

Figure 3: Route of the logistics train and its sensors for stopping.
100 pieces. The towing vehicle and transporters had to be created as new entities. The speed of the train is $1 \mathrm{~m} \cdot \mathrm{s}^{-1}$.

\subsection{Sub-model production}

The main production operations take place in this part of the simulation model (Figure 4). The main production operations include the machining of components and their subsequent assembly. After the complete assembly of gearbox, the next step is its testing. The final products are then transported by a logistics train to the output store.

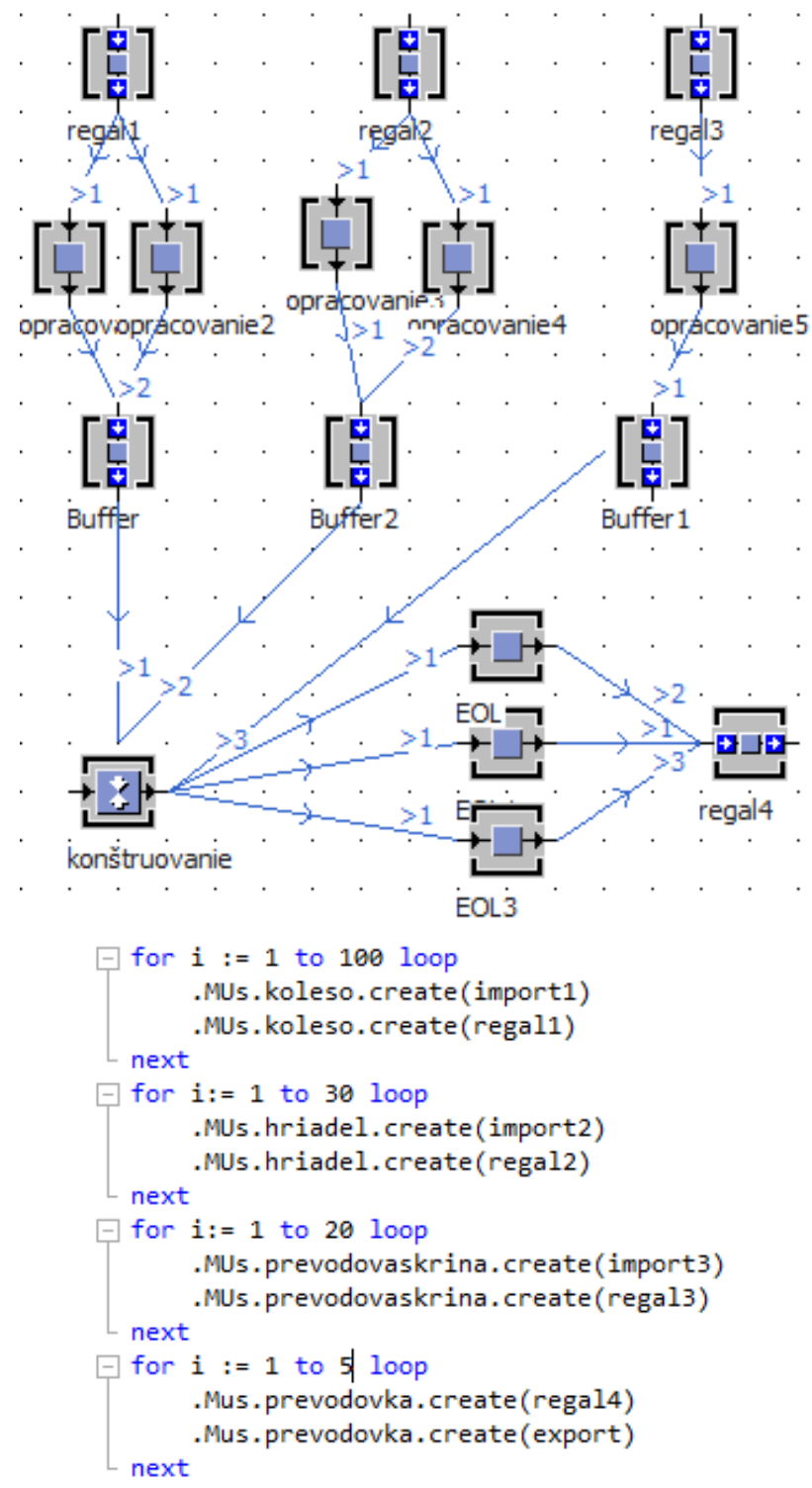

Figure 4: Demonstration of the production process and method of entities generation 


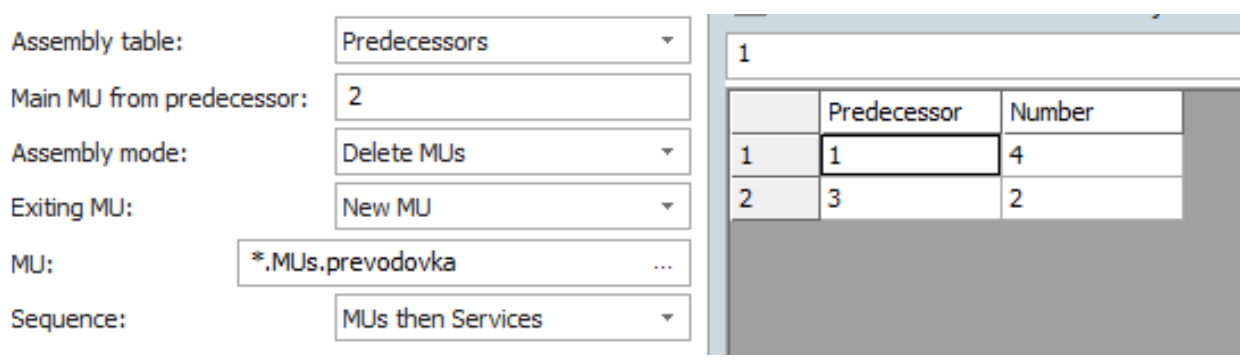

Figure 5: Setting of the block Assembly

The creation of individual entities in the shelves and in the input store is realized by the method (Figure 4). The created entities are passive elements that cannot perform activities or movements. Therefore, it is necessary for their transport to use for example workers or transport means. At the beginning of simulation, it is needed to define the initial states of the entities, so that after the simulation starts production to be able to produce.

Subsequently, the components are machined at different times, the processing time for the wheels is 45 second, the processing time for shafts is 180 second and the processing time for gear-boxes is 85 seconds. After the machining of the component, it is necessary to pre-store them. It was used the block buffer for their placing. This block may not only present a store, but it may also present an area, a free space, a shelf, store, and others. In this block, components can be stored for a specified time. The components are machined at different processing times and therefore it is needed to store them in a certain location.

Blocks are connected by connectors. The block Assembly is used for the assembly of the components. The table for assembly (Figure 5) is set in a way that from the predecessor 1 is a required number of entities 4 . From the predecessor 3 is needed a number of entities 2 . The main predecessor is 1 . By setting off the main predecessor, it is removed the number of entities in number 1 . By connection of the required entities that present all components, a new entity is created and this presents the assembled gearbox. The gearbox is further moved to blocks endofline1 and endofline2. These blocks present the final check. Consequently, the finished products may leave the production. They wait for loading to the logistics train in the block shelf 2.

\subsection{Output store}

The final part of the simulation model is the output store with finished products (Figure 6).

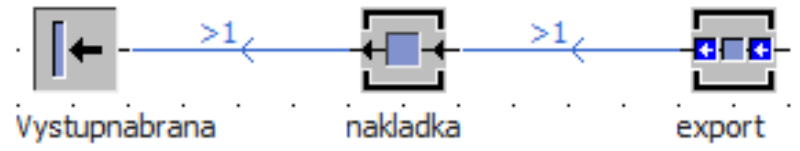

Figure 6: Process of the output store

In the output store, the products are reloaded from the train to the block Buffer, specifically export, i.e. to a certain place (store, shelf), from which they are subsequently loaded and delivered.

\subsection{Use of the system Kanban in the simulation model}

The supply of entities from the input store to the production is controlled by the principle of the system Kanban. Entities are moved on the logistics train in the number required for production. After input of the entity to the production, the requirement for delivery of the entities to the production is deducted. Subsequently after entity machining, in the buffer, the requirement for suppliers of entities to production is added and sent to the input store, where by the requirements of the production, the entities are transported to the logistics chain. For the given requirements, in the simulation model were created methods with a specific statement.

The statement for deduction of the input entity of wheels to the production is as follows $\mathrm{A}$ : $=\mathrm{A}-1$. For deduction of the entities of shafts is the statement $\mathrm{B}:=\mathrm{B}-1$ and for gearboxes $C:=C-1$. Requirements for supplies from the input store. The methods for adding entities are specified by output from the blocks Buffer before assembly. For the wheels $A:=A+1$, shafts $B:=B+1$ and gearboxes $C:=C+1$. Presentation of requirements changes depending on the produced products. After the first run of the logistics train, the requirements are loaded to the logistics train. Requirements can be sent electronically by scanning of $Q R$ codes from entities. The scanned data are sent to the 

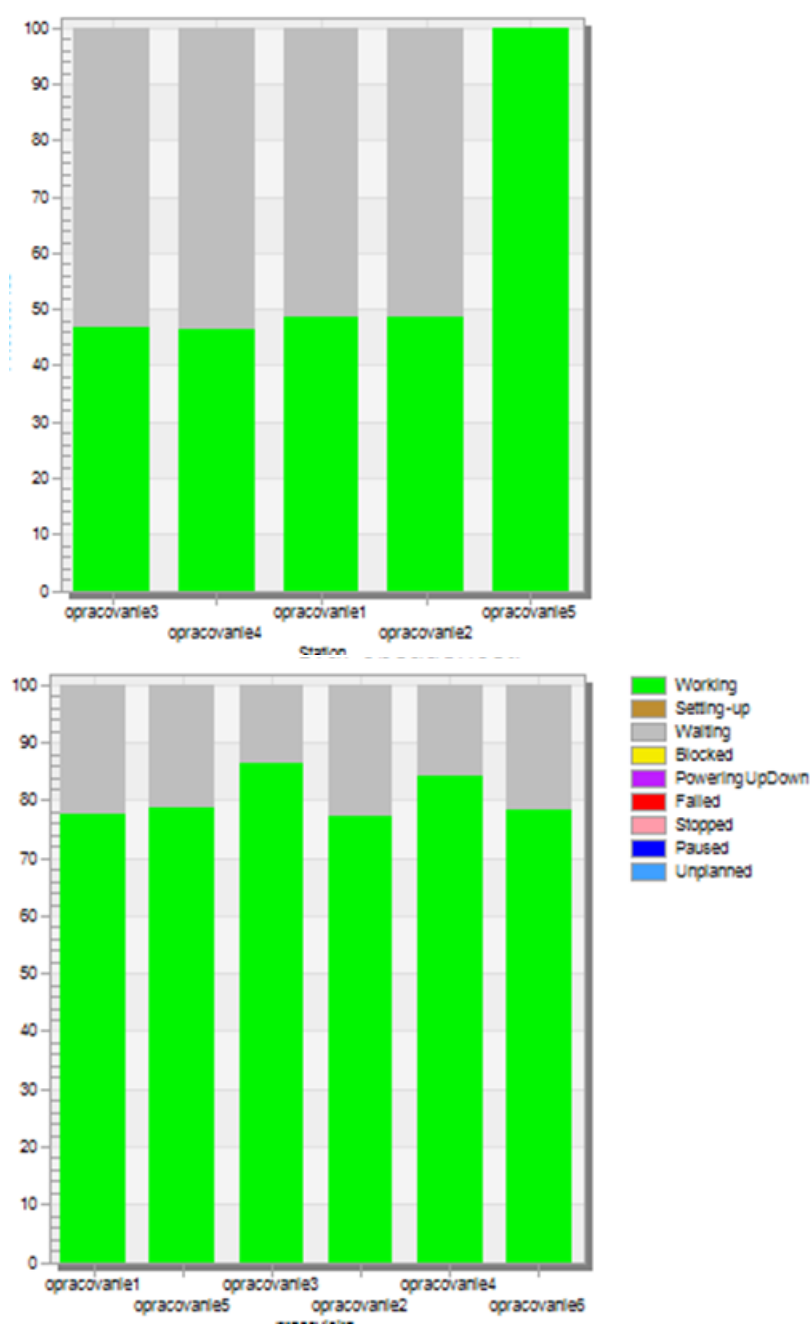

Figure 7: Graph of utilization before modification and after modification

input store. This system prevents the formation of various anomalies that can occur with the classic card Kanban.

\section{Results}

For the need of intra-plant transport operation planning by AGV simulation experiments were realized with the model. Within the real process of supply, various anomalies occurred that had to be eliminated and optimized. All workplace, stores and objects were monitored by graphs.

One of the accrued anomalies was insufficient utilization of workplaces, machining and an insufficient amount of input stocks. Increase of workplace utilization was achieved by increasing the number of input supply in shelves. Initially, an amount of 10 was added from each en-
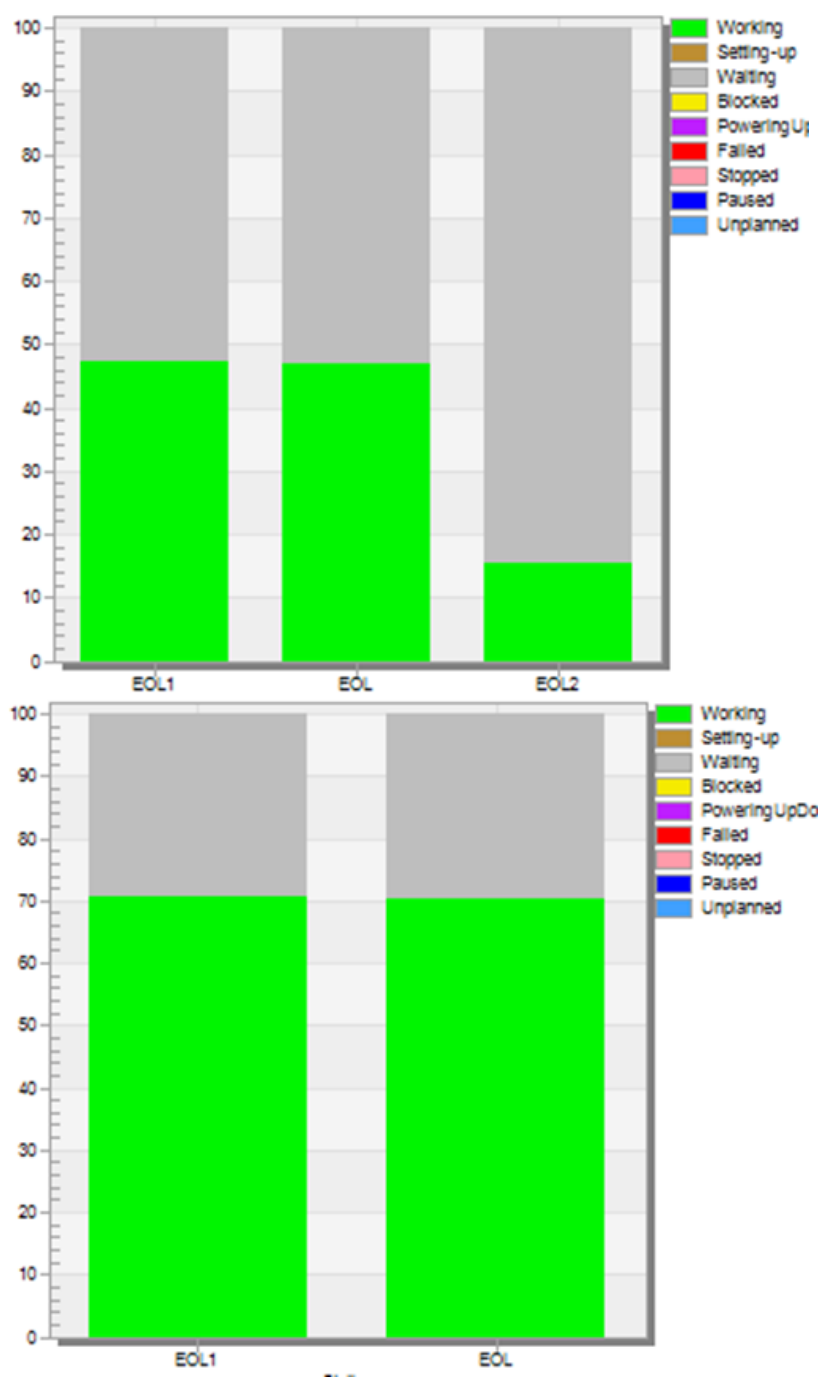

Figure 8: Graph of the utilization of EOL before the change and after the change

tity, but the utilization of workplaces changed minimally. The next step was to increase the number of entities by 30 . In this case, the utility of workplaces increased and utility was not under $70 \%$. The graph (Figure 7 ) presents that the utility of the workplace was not higher than $50 \%$ and adding of initial input entities increased the utility of workplace to more than $70 \%$ (Figure 7 ).

The graphs also present that the workplace with the title machining 5 is maximally utilize compared to another workplace. The workplace machining 5 did not manage machining the entities and blocking assembly. The next simulation experiment was adding workplace for machining of entities from the shelf 3 , adding a workstation the production run more smoothly and not block assembly. It is not necessary to achieve maximal utility at workplaces 
because after machining is the process of assembly which is sufficiently supplied.

By the help of the graphs in the monitored simulation model, it was found that the utilization of workplaces is not optimal. The workplace EOL2 is utilized at approximately $10 \%$ (Figure 8). The suitable solution for optimization of workplaces was removal the third workplace EOL2. After the removal of the third workplace EOL2, it was achieved the better utilization of the workplaces EOL and EOL1 (Figure 8). The workplaces work after these changes at about $70 \%$.

\section{Conclusion}

Planning of intra-plant transport operation presents a challenging process that needs to reflect different types of criteria and parameters. Nowadays, by reason of the complexity and scale of logistics systems, it is needed to use simulation approaches that are realized by functional simulation tools. On the part of achievement the details of the modelled processes, it is particularly desirable, that these tools dispose of considerable variability and customization. These tools are also presented by the application of the method of additive programming. This helps to create simulation models for the need for detailed analysis of individual processes, identification of critical points and finding of optimal solutions.

The paper by the presented example points out to the fact that this type of simulation model can increase the performance of a complex logistics system by up to $70 \%$ and reduce the number of workplaces, in this case by one, by using the additional programming method. The paper deals with the previous research of authors which was focused on the possibility of applying the method of additional programing and its direct use in the field of intraplant transport system using AGV. In the future, the research is focused on examination of possible variability of models, automating scripting in the program SimTalk and accelerating the creation of reliable and valid simulation models. The aim of the research will be an overall acceleration of the whole process which this method can implement in practice.

Acknowledgement: This work is a part of these projects VEGA 1/0063/16, VEGA 1/0638/19, KEGA 012TUKE-4/2019 and APVV SK-SRB-18-0053.

\section{References}

[1] Gola, A. \& Klosowski G. (2019). Development of computercontrolled material handling model by means of fuzzy logic and genetic algorithms. Neurocomputing. 338(21), 381-392. DOI: 10.1016/j.neucom.2018.05.125.

[2] Digani, V. Hsieh, MA. Sabattini, L. \& Secchi, C. (2019). Coordination of multiple AGVs: a quadratic optimization method. Auton Robots. 43(3), 539-555. DOI: 10.1007/s10514-018-9730-9.

[3] Osman, K. Ghommam, J. Saad, M. (2018). Optimal Path Planning and Tracking for AGV Lane Merging. 2018 15TH Int. MULTICONFERENCE Syst. SIGNALS DEVICES, 345 E 47TH ST, NEW YORK, NY 10017 USA: IEEE, 19-22 March 2018 (402-407).

[4] Tavakoli, MM. Haleh, H. Mohammadi, M. (2018). A mathematical model for scheduling of production process and allocation of an automatic guided vehicle in a flexible manufacturing system. Int J Eng Syst Model Simul 2018: IEEE, 19-22 March 2018 (125-131).

[5] Varecha, D. Kohar, R. \& Brumercik, F. (2019). AGV Brake System Simulation, LOGI - Scientific Journal on Transport and Logistics, 10(1), 1-9. DOI: 10.2478/logi-2019-0001

[6] Fazlollahtabar, H. \& Saidi-Mehrabad (2015). Optimal path in an intelligent AGV-based manufacturing system. Transp Lett Int J Transp Res 2015. 7(4), 219-228. DOI: 10.1179/1942787514Y.0000000047.

[7] Conkur, ES. (2005). Path planning using potential fields for highly redundant manipulators. Rob Auton Syst 2005. 52(2-3), 209-228. DOI: 10.1016/j.robot.2005.03.005.

[8] Janekova, J., Fabianova, J., Izarikova, G., Onofrejova, D., \& Kovac, J., (2018). Product mix optimization based on monte carlo simulation: a case study. Int J Simul Model. 17(2), 295-307. DOI: 10.2507/IJSIMM17(2)436.

[9] Mikusova, N. Cujan, Z. Tomkova, E. (2017). Robotization of logistics processes. In 18th International scientific conference-LOGI 2017, 19 October 2017 (pp. 1-8). Ceske Budejovice, Czech republic: EDP Sciences.

[10] Molnar, V. \& Pacutova, K. (2001). Assessing the possibilities of reducing the transport company costs. In Proceedings of the third international conference on traffic and transport engineering (ICTTE), 24-25 November 2016 (pp. 570-575). Belgrade, Serbia: Scientific research center LTD Belgrade.

[11] Stopka, O. Kampf, R. Kolar J. \& Kubasakova I. (2014). Identification of Appropriate Methods for Allocation Tasks of Logistics Objects in a Certain Area. Nase More. 61(1-2), 1-6.

[12] Fedorko, G., Molnar, V., Vasil, M., \& Hanzl, J., (2018). Application of the Tecnomatix Plant Simulation Program to modelling the handling of ocean containers using the AGV system. N. More. 65(4), 230-236. DOI: 10.17818/NM/2018/4SI.12.

[13] Neradilova, H. Fedorko, G. \& Borsosova, V. (2008). Modelling of intra plant transportation system using AGV. In The third International conference on traffic and transport engineering (ICTTE), November 2016 (pp. 971-979). Belgrade, Serbia: Scientific research center Ltd Belgrade.

[14] Cesarotti, V. Di Silvio, N. Introna, V. \& Mori, G. (2016). Evaluation and optimisation of manufacturing system using simulation modelling and design of experiment. In EMSS 2008: 20th European modeling and simulation symposium, 17-19 September 2008 (pp. 224-230). Amantea, Italy: Diptim-Univ Genoa.

[15] Trebuna, P., Pekarcikova, M., \& Edl, M., (2019). Digital value stream mapping using the Tecnomatix Plant Simulation software. 
Int. J. of Sim. Model. 18(1), 19-32. DOI: 10.2507/IJSIMM18(1)455.

[16] Petrila, S. Brable, G. \& Chirita, B. (2016). The use of Tecnomatix software to simulate the manufacturing flows in an industrial enterprise producing hydrostatic components. In Modtech international conference - Modern technologies in industrial engineering IV, PTS 1-7, $15-18$ June 2016 (pp. 1-9). Iasi, Romania: IOP publishing LTD, Bristol, England. 